\title{
Genes with stable DNA methylation levels show higher evolutionary conservation than genes with fluctuant DNA methylation levels
}

\author{
Ruijie Zhang ${ }^{1, *}$, Wenhua Lv ${ }^{1, *}$, Meiwei Luan ${ }^{1, *}$, Jiajia Zheng ${ }^{1, *}$, Miao Shi, ${ }^{1,}$, Hongjie \\ Zhu $^{1}$, Jin Li $^{1}$, Hongchao Lv ${ }^{1}$, Mingming Zhang ${ }^{1}$, Zhenwei Shang ${ }^{1}$, Lian Duan ${ }^{1}$, \\ Yongshuai Jiang ${ }^{1}$ \\ ${ }^{1}$ College of Bioinformatics Science and Technology, Harbin Medical University, Harbin 150086, China \\ *These authors have contributed equally to this work \\ Correspondence to: \\ Yongshuai Jiang, e-mail: jiangyongshuai@gmail.com \\ Ruijie Zhang, e-mail: zhangruijie2013@gmail.com \\ Keywords: DNA methylation stability, evolutionary characteristics, SNP density, linkage disequilibrium \\ Received: July 26, $2015 \quad$ Accepted: October 06, $2015 \quad$ Published: October 19, 2015
}

\section{ABSTRACT}

Different human genes often exhibit different degrees of stability in their DNA methylation levels between tissues, samples or cell types. This may be related to the evolution of human genome. Thus, we compared the evolutionary conservation between two types of genes: genes with stable DNA methylation levels (SM genes) and genes with fluctuant DNA methylation levels (FM genes). For long-term evolutionary characteristics between species, we compared the percentage of the orthologous genes, evolutionary rate $\mathrm{dn} / \mathrm{ds}$ and protein sequence identity. We found that the SM genes had greater percentages of the orthologous genes, lower dn/ds, and higher protein sequence identities in all the 21 species. These results indicated that the SM genes were more evolutionarily conserved than the FM genes. For short-term evolutionary characteristics among human populations, we compared the single nucleotide polymorphism (SNP) density, and the linkage disequilibrium (LD) degree in HapMap populations and 1000 genomes project populations. We observed that the SM genes had lower SNP densities, and higher degrees of LD in all the 11 HapMap populations and 131000 genomes project populations. These results mean that the SM genes had more stable chromosome genetic structures, and were more conserved than the FM genes.

\section{INTRODUCTION}

DNA methylation is an epigenetic mechanism $[1,2]$ that plays important roles in the regulation of gene expression [3, 4], development [5-7], X-chromosome inactivation [8] and genomic imprinting $[9,10]$.

Some genomic regions can exhibit different methylation statuses among multiple samples (tissues, cell types or individuals) [11]. Differences in DNA methylation levels can identify a methylation locus associated with susceptibility to diseases, such as Alzheimer's disease $[12,13]$, cardiovascular disease $[14,15]$, and cancer $[16,17]$. Other genomic regions may exhibit robust or stable methylation statuses between different tissues, samples or cell types.
To explore the genetic basis of maintaining DNA methylation levels, we investigated the relationship between evolutionary conservation and the stability of DNA methylation levels. In this study, two types of genes were considered: SM genes were defined as genes that have stable DNA methylation levels in all cell types of every tissue under normal or disease conditions. In other words, the DNA methylation statuses of SM genes were robust to environmental changes. FM genes were defined as genes that have fluctuant DNA methylation levels in different cell types, tissues, organisms or samples; i.e., FM genes were sensitive to environmental changes. For SM genes and FM genes, we compared the long-term evolutionary characteristics and short-term evolutionary characteristics. We found a strong association between 
the stability of DNA methylation levels and evolutionary conservation.

\section{RESULTS}

To make the results more robust, we used a unified platform (GPL13534) and a large sample size (8,676 samples). For each of the 21,231 genes, we employed a fluctuation coefficient, FC, to measure the stability of DNA methylation levels. We then constructed two gene sets, SM genes (4,247 genes) and FM genes $(4,247$ genes), and compared their differences in evolutionary conservation.

\section{SM genes had a higher percentage of orthologous genes than FM genes}

For SM and FM genes, we calculated the numbers of the one-to-one orthologous genes in each of the 21 species. The results are displayed as a bar chart in Figure 1, which shows that the bar chart of the SM genes is higher than that of the FM genes. We also used the Wilcoxon signed rank test to test the statistical significance. The null hypothesis is that the SM genes and the FM genes had the same percentage of orthologous genes in each of the 21 species. The $P$-value was $9.54 \mathrm{E}-07$ and the significance level $\alpha$ was 0.05 . This indicated that the SM genes had a higher percentage of orthologous genes than FM genes across the 21 species.

\section{SM genes had a lower evolutionary rate than FM genes}

For each of the 21 species, we calculated the evolutionary rate $\mathrm{dn} / \mathrm{ds}$ for orthologous genes of the SM and FM genes. The bar chart of the SM genes is lower than that of the FM genes (Figure 2A). A Wilcoxon signed rank test gave a significant $P$-value $(P=6.41 \mathrm{E}-05)$. Thus, the SM genes had lower dn/ds than the FM genes across all species. For each species, we also drew a box plot of the SM genes against the FM genes (Figure 2B). A Wilcoxon rank sum test produced significant $P$-values for each of the 21 species (for details, see Supplementary Table S1). These results indicated that the SM genes had a lower evolutionary rate than the FM genes.

\section{SM genes had a higher protein sequence identity than FM genes}

We aligned the orthologous proteins between human and the other 21 species using BLASTP software. For SM and FM genes, we extracted the sequence identity from the alignment results. Comparing the medians (Figure 3A) showed that the SM genes had a higher protein sequence identity across all species (Wilcoxon signed rank test, $P=6.41 \mathrm{E}-05)$. Furthermore, we also compared the protein sequence identity between SM genes and FM genes in each of the 21 species. The protein sequence identity of SM genes was significantly higher than that of the FM

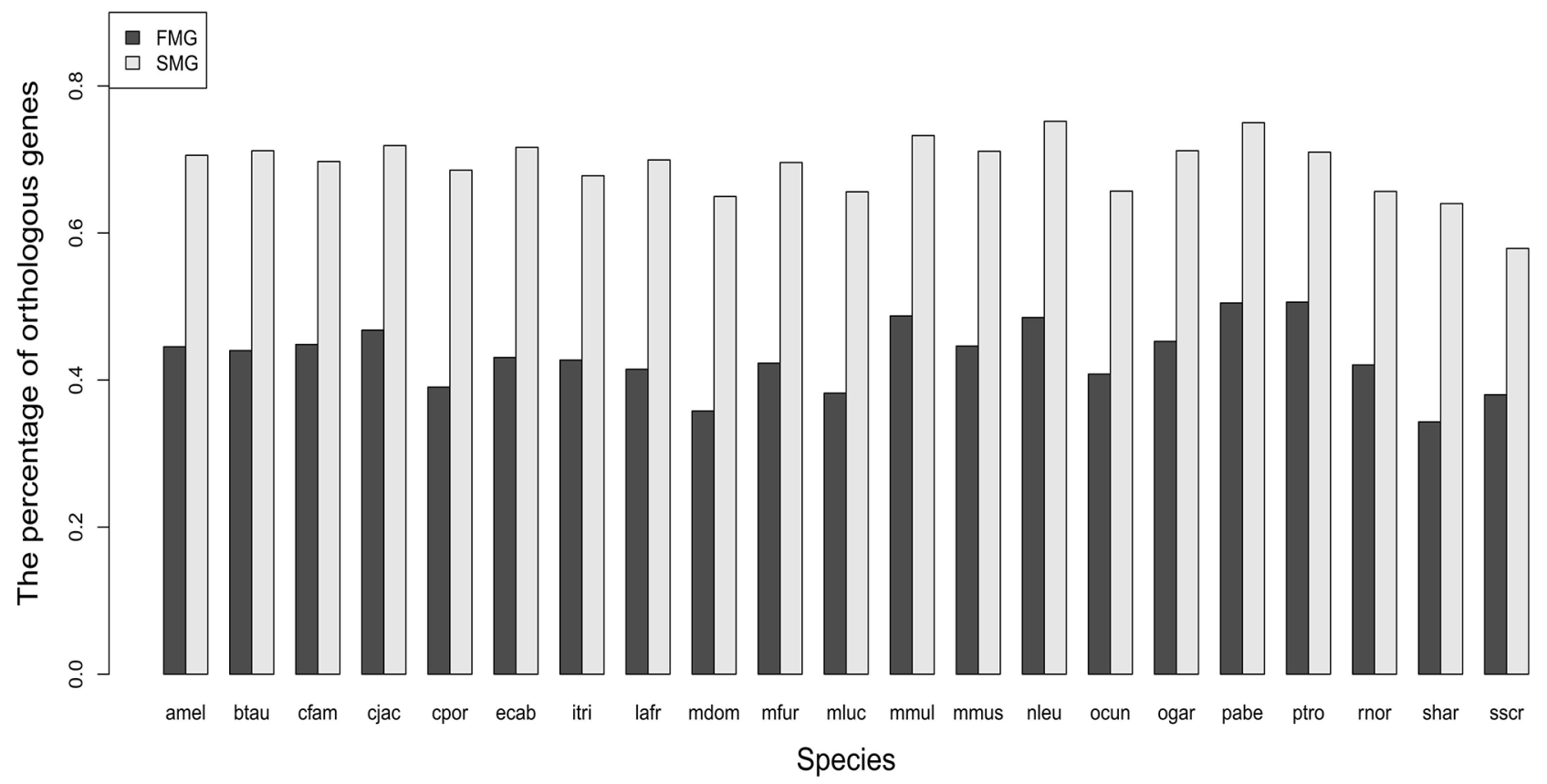

Figure 1: A bar chart comparing the percentage of orthologous genes between SM and FM genes. 


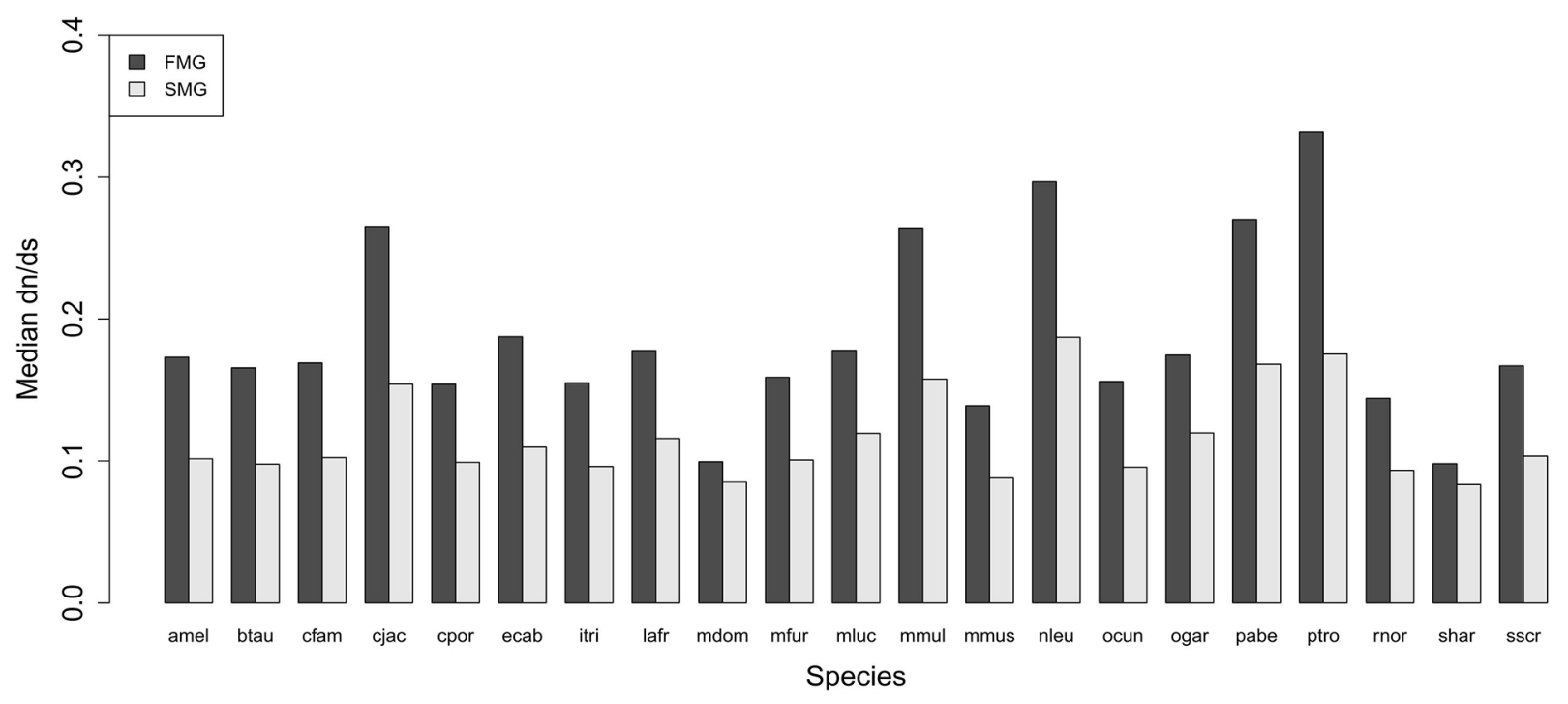

(B)
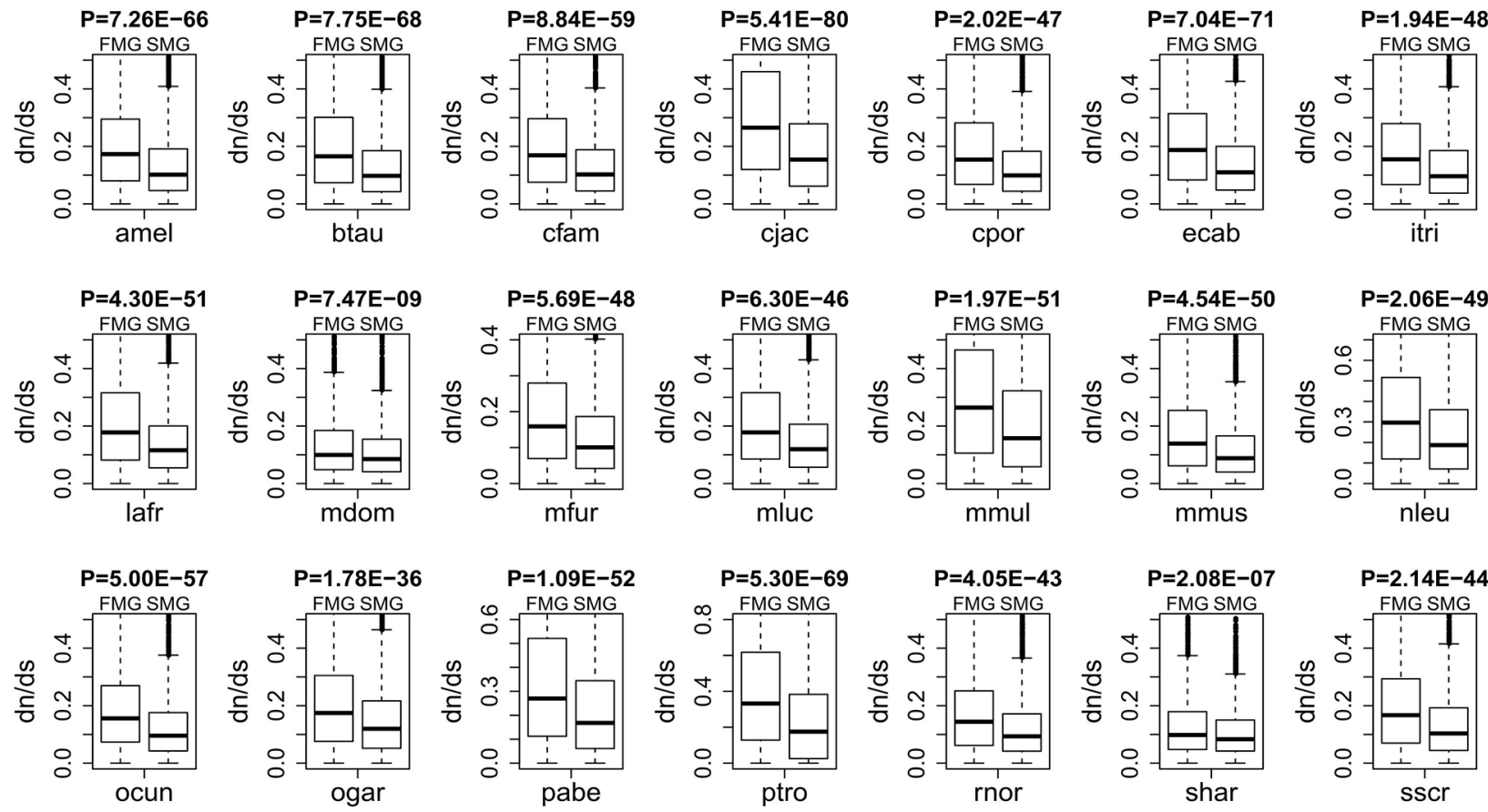

Figure 2: Comparison of the evolutionary rate, dn/ds, between SM and FM genes. A. A bar chart of the SM genes against the FM genes. B. Box plots of the SM genes against the FM genes for each of the 21 species.

genes for each species (Figure 3B). This also indicated that the SM genes were more conserved than the FM genes. Supplementary Table S2 showed the detailed information about the sequence identity for each species.

\section{SM genes had a lower SNP density than FM genes}

We also compared some short-term evolutionary characteristics of the SM and FM genes. In the human genome, the most common single base genetic variation is the SNP $[18,19]$. In this study, the SNP density in a gene region was used to measure the degree of genetic variation. The FM genes had higher SNP densities than the SM genes (Figure 4). A Wilcoxon rank sum test produced a significant $P$-value $(P<3.54 \mathrm{E}-16)$. This indicated that the FM genes contained significantly more genetic variations than the SM genes, i.e., the SM genes were more conservative. 


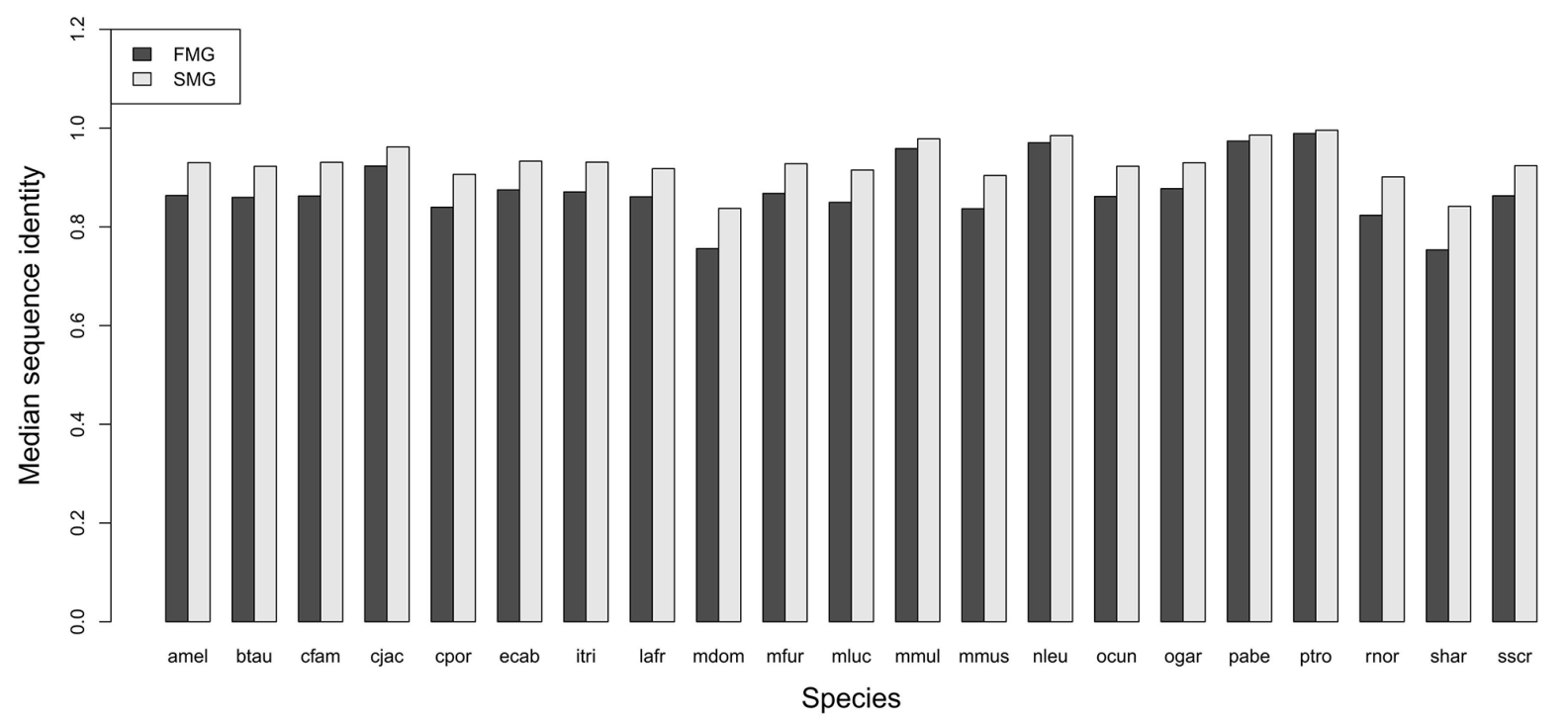

(B)
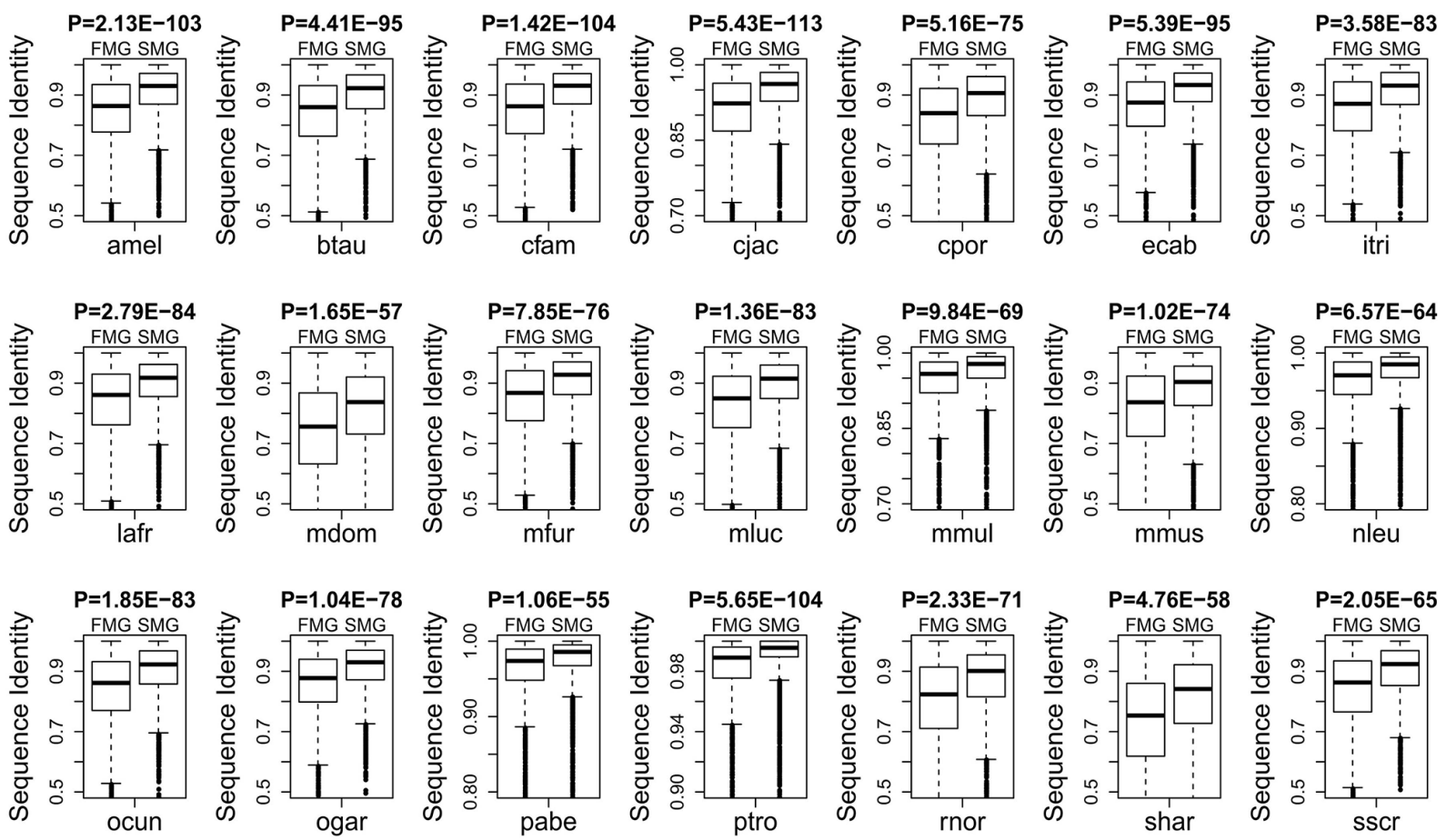

Figure 3: Comparison of the sequence identity between SM and FM genes. A. A bar chart of the SM genes against the FM genes. B. Box plots of the SM genes against the FM genes for each of the 21 species.

\section{SM genes had a higher degree of LD in all 11 HapMap populations and 131000 genomes populations}

For each of the 11 HapMap and 131000 genomes populations, we calculated the LD coefficient, $\mathrm{r}^{2}$, for the SM and FM genes. We also drew a bar chart of the median $\mathrm{r}^{2}$ of the SM genes against the FM genes across all the HapMap populations (Figure 5A) and all the 1000 genomes populations (Figure 6A). Whether in HapMap populations or in 1000 genome populations, the bar chart of the SM genes was higher than that of the FM genes. Figure 5B and Figure 6B show that the $P$-values were significant for all the 11 HapMap populations and 


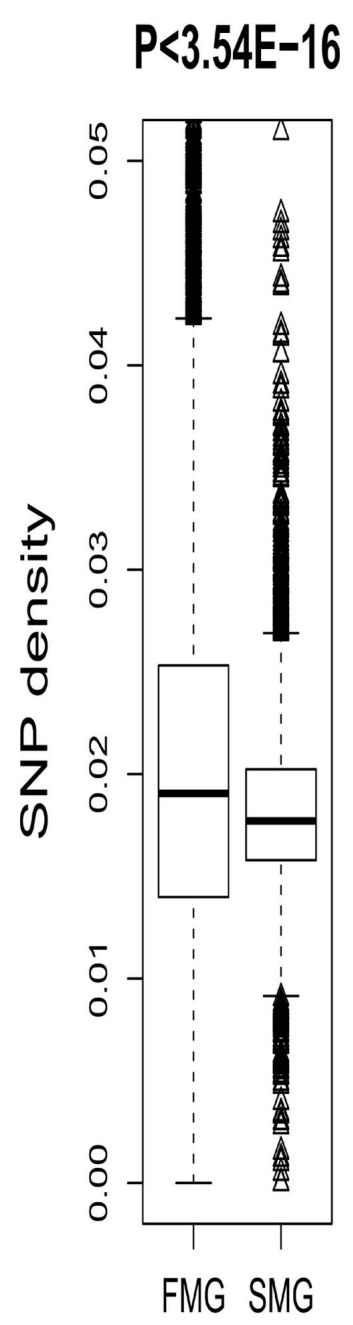

Figure 4: A box plot comparing the SNP density between SM and FM genes.

all the 131000 genomes populations. For details, see Supplementary Table S3 (the median, upper and lower quartiles of $\mathrm{r}^{2}$ for HapMap populations) and Supplementary Table S4 (the median, upper and lower quartiles of LD coefficient $r^{2}$ for 1000 genomes populations). Thus, the SM genes had a higher degree of LD than the FM genes. Compared with FM genes, SM genes had undergone fewer recombination events, and had more stable chromosome genetic structures, i.e., the SM genes were more conserved.

\section{SM genes had different biological functions comparing with FM genes}

We found that, for biological process (BP), SM genes were mainly annotated to certain metabolic processes that are critical to growth, reproduction and maintenance of structures and survival of cells, such as cellular metabolic process (GO:0044237, BP), cellular macromolecule metabolic process (GO:0044260, BP), metabolic process (GO:0008152, BP), macromolecule metabolic process (GO:0043170, BP), and primary metabolic process (GO:0044238, BP). For molecular function (MF), SM genes were mainly annotated to certain basic binding categories, such as nucleic acid binding (GO: 0003676, MF), RNA binding (GO: 0003723 , MF) and binding (GO: 0005488, MF). These processes or functions are important for maintaining the stability of cells or organisms. FM genes were mainly annotated to processes (BP) or functions (MF) that interact with the environment, such as sensory perception of smell (GO: 0007608, BP), sensory perception of chemical stimulus (GO: 0007606, BP), G-protein coupled receptor protein signaling pathway (GO: 0007186, BP), sensory perception (GO: 0007600, BP) cognition (GO: 0050890, BP), olfactory receptor activity (GO: 0004984), G-protein coupled receptor activity (GO: 0004930, MF), transmembrane receptor activity (GO: 0004888, MF), receptor activity (GO: 0004872, MF) and molecular transducer activity (GO: 0060089, MF). These annotation results hint at the biological reasons underlying the differences in evolutionary conservation. Genes that maintain the basic survival of the cell tend to have stable 
(A)

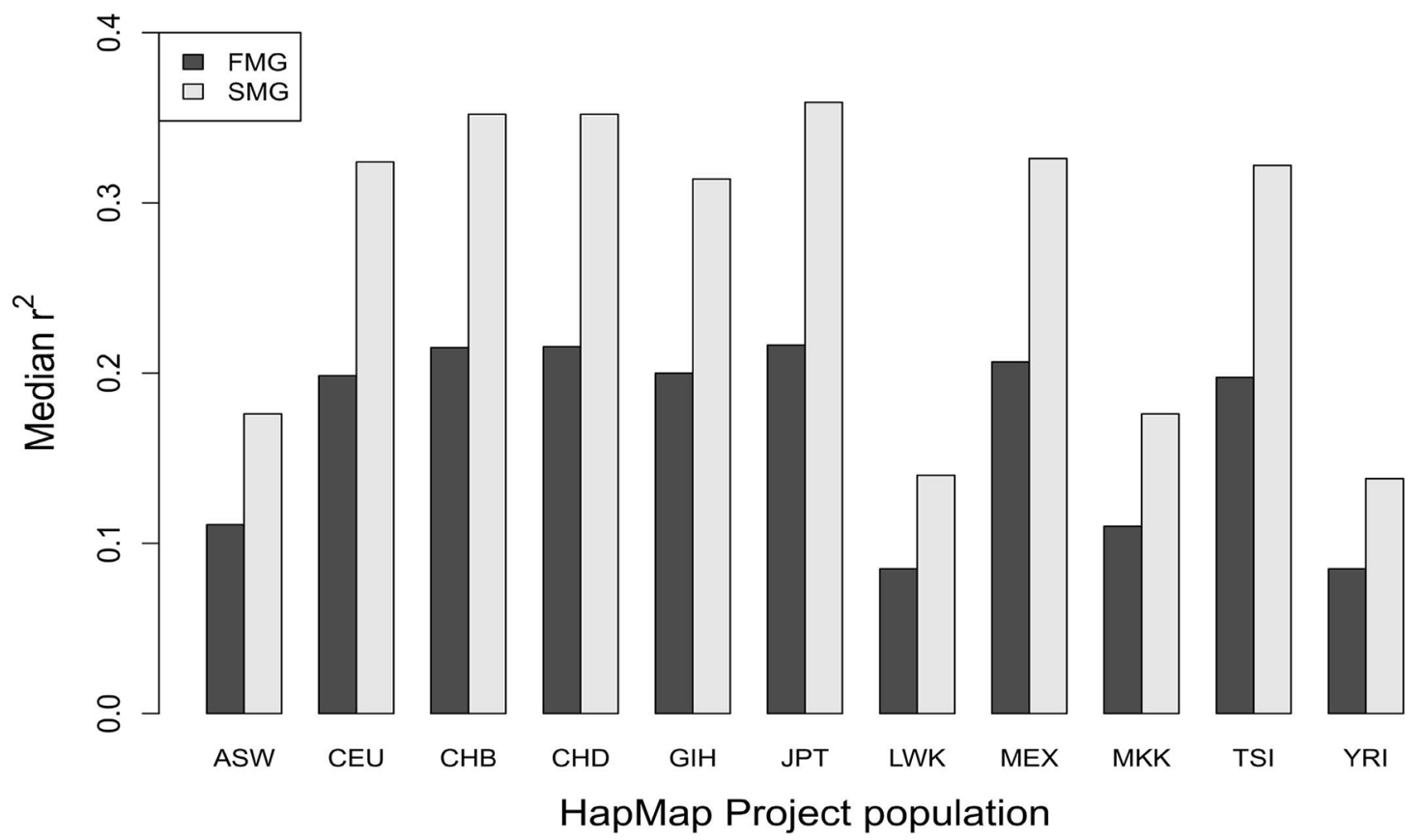

(B)
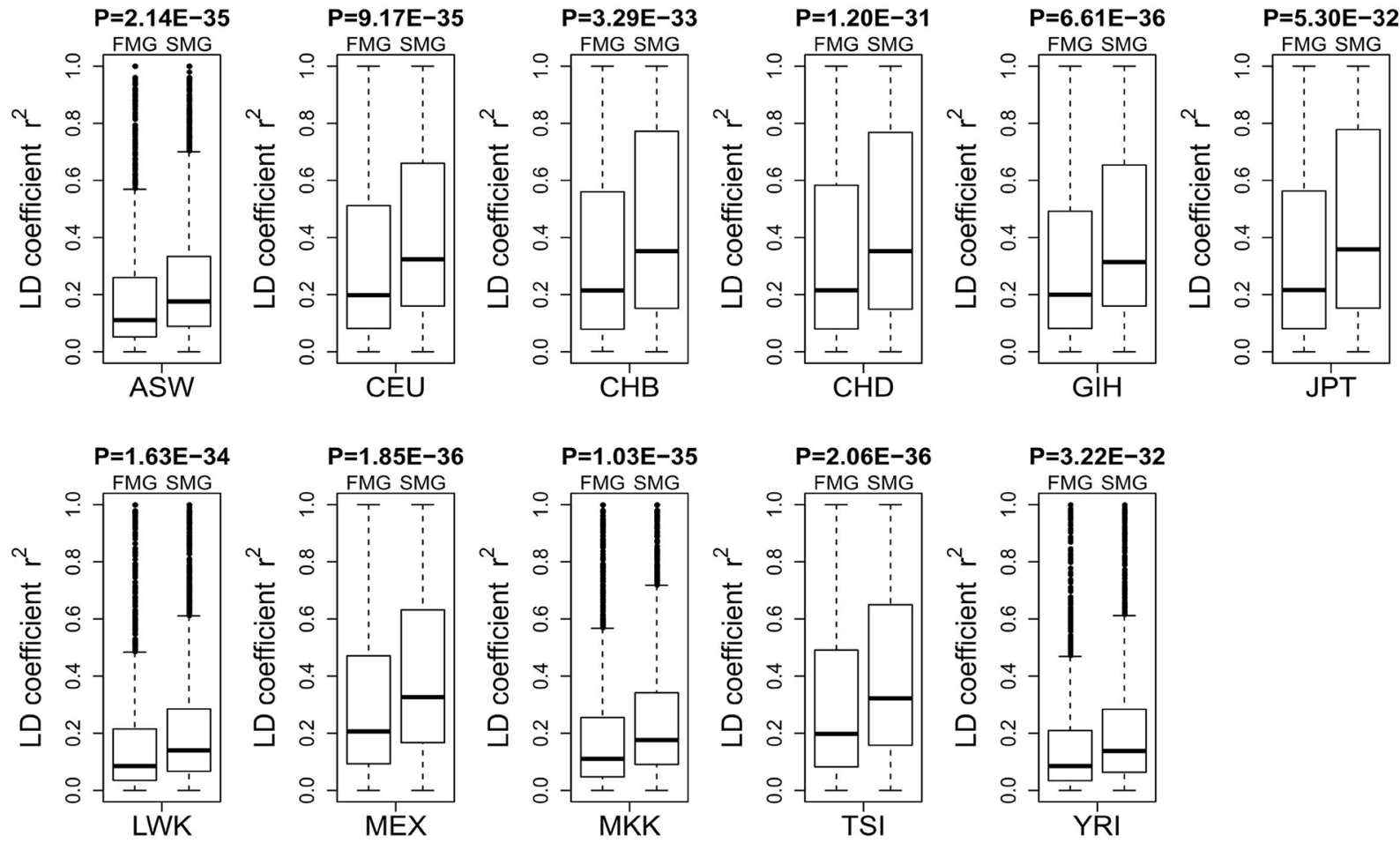

Figure 5: Comparison of the $\mathbf{r}^{2}$ value between SM and FM genes. A. A bar chart of the SM genes against the FM genes. B. Box plots of the SM genes against the FM genes for each of the 11 HapMap populations. 
(A)

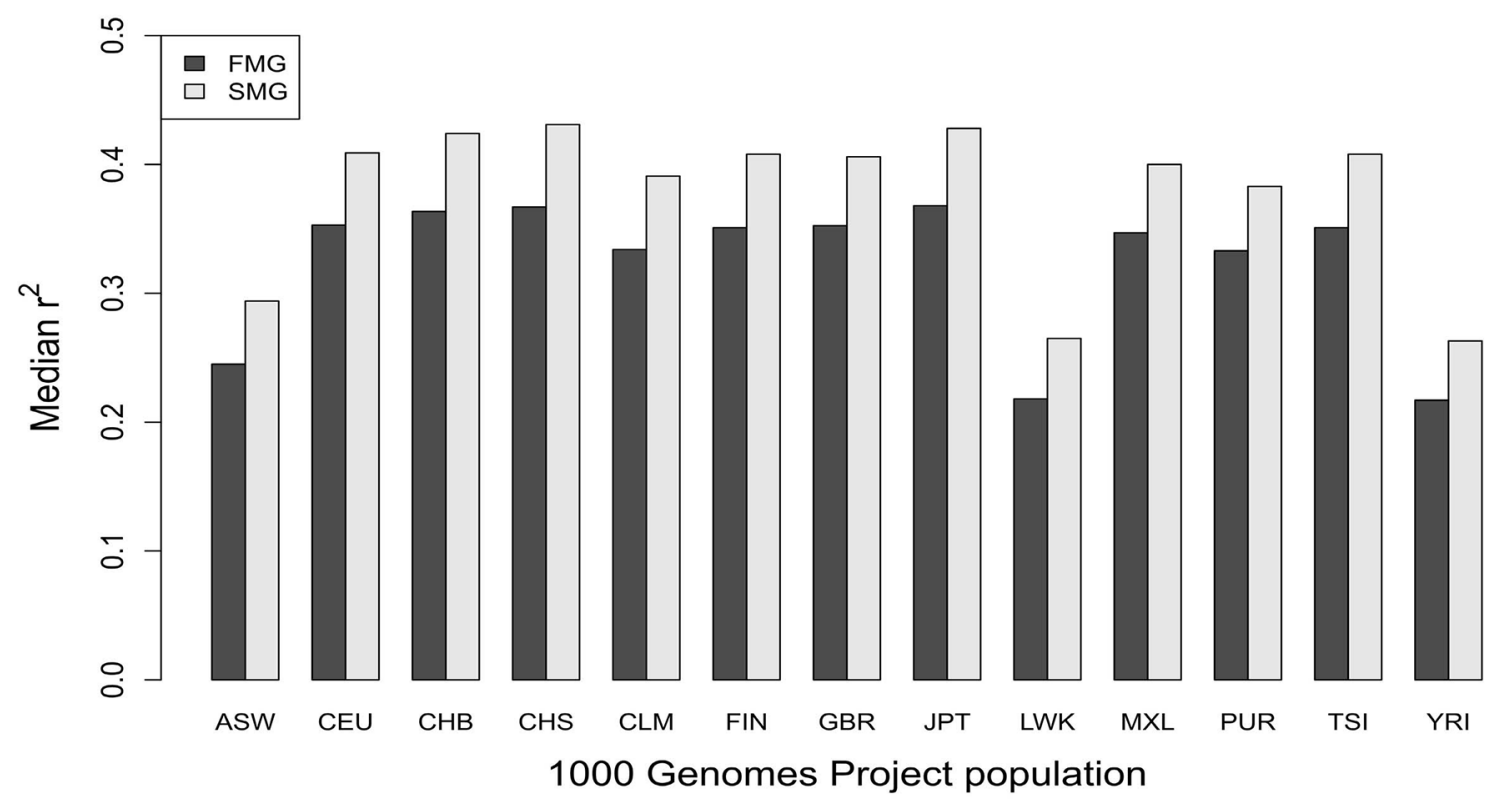

(B)
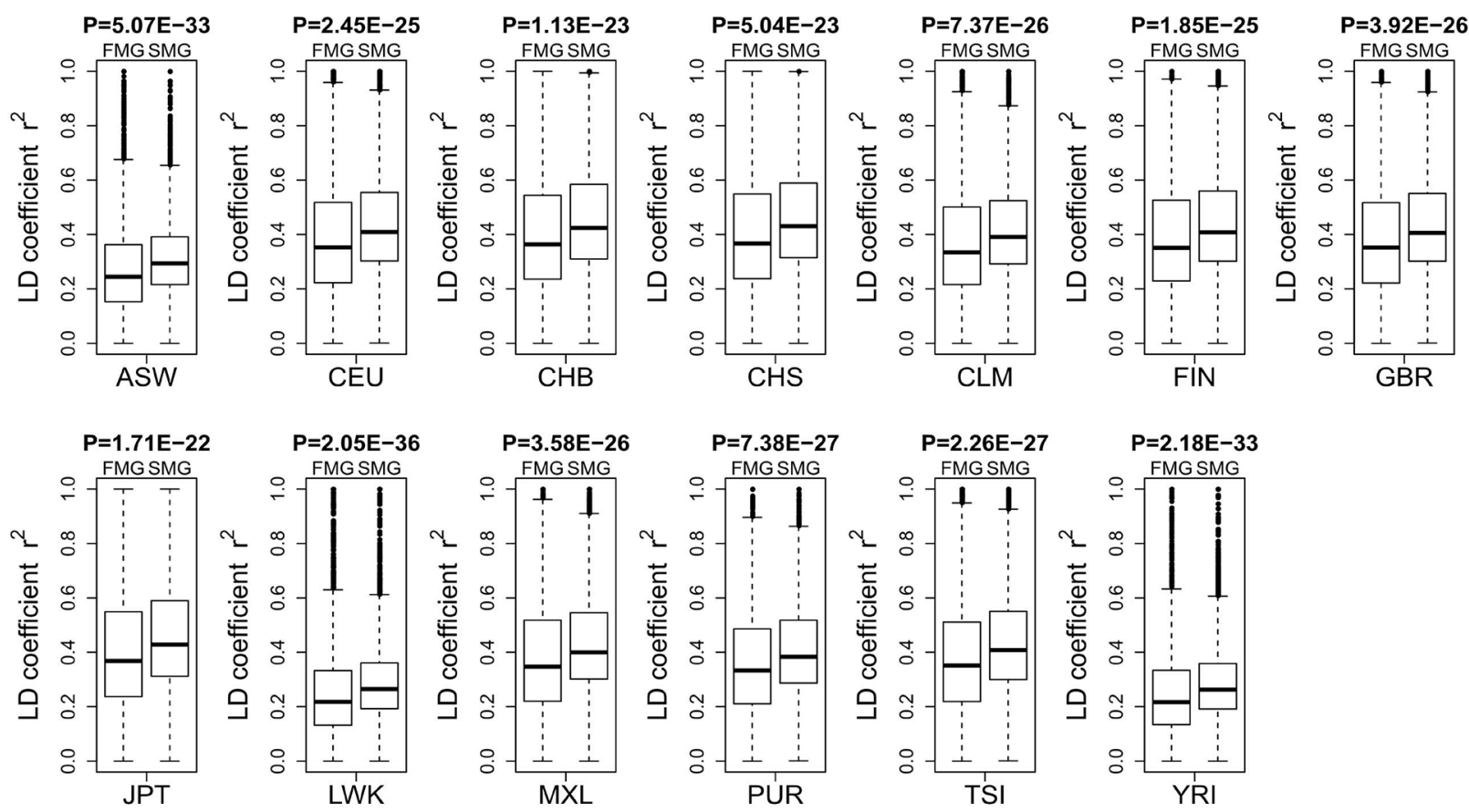

Figure 6: Comparison of the $\mathbf{r}^{2}$ value between SM and FM genes. A. A bar chart of the SM genes against the FM genes. B. Box plots of the SM genes against the FM genes for each of the 131000 genomes project populations.

DNA methylation levels. For details, see Supplementary File Section 2 (the GO annotation results of SM genes, from Supplementary Table $2-1$ to Supplementary Table 2-3) and Supplementary File Section 3(the GO annotation results of FM genes, from Supplementary Table 3-1 to Supplementary Table 3-3).

\section{DISCUSSION}

To ensure the reliability of the results, we first chose a large sample set (8,676 samples) to identify the SM and FM genes. Then we compared three long-term (species) evolutionary characteristics (the percentage 
of the orthologous genes, evolutionary rate $(\mathrm{dn} / \mathrm{ds})$ and protein sequence identity) and two short-term (human) evolutionary characteristics (the SNP density and LD degree) between the SM and FM genes. We came to the following six conclusions: (1) the SM genes had a greater percentage of the orthologous genes than FM genes for all the 21 species; (2) the SM genes had a lower evolutionary rate than the FM genes for all 21 species; (3) the SM genes had a higher protein sequence identity than FM genes for all 21 species; (4) the SM genes had a lower SNP densities than the FM genes; (5) the SM genes had a higher degree of LD than the FM genes for all 11 HapMap populations; and (6) the SM genes had a higher degree of LD than the FM genes for all 131000 genomes populations. These conclusions supported the hypothesis that the SM genes were more evolutionarily conserved than the FM genes.

Furthermore, we also compared the functional difference between the SM and FM genes using the GO database. The BP and MF categories were considered. The comparison showed that SM genes were mainly annotated to metabolic processes maintaining the basic survival of the cell, while FM genes were mainly annotated to processes or functions interacting with the environment, such as sensory perception of smell and sensory perception of chemical stimulus. A broad definition for metabolism is the sum of the biochemical processes of living organisms [20]. Metabolism performs a fundamental role in biology and impacts almost all functions of cells [21]. Many studies have demonstrated the relationship between metabolism and the pathogenesis of disease [20, 22, 23], implying that the SM genes involved in metabolic processes play crucial roles in the genome. These genes might have stable genomic structures, and undergo few mutations and recombination events during evolution. Compared with the SM genes, the FM genes showed less evolutionary conservation in both long-term evolutionary features and short-term evolutionary features. They were mainly involved in the response to environmental stimuli, implying that the FM genes exhibit less genomic stability and evolve faster during evolution to allow adaptation to changes in the environment.

To test whether the results of our study were strong and reliable, we also extracted top (bottom) $15 \%$ and top (bottom) 25\% genes (as ranked by FC values) and investigated the difference of evolutionary features for the two extreme gene sets. We found that the results of $15 \%$ and $25 \%$ are consistent with that of top and bottom $20 \%$. The results for the top (bottom) 15\% and top (bottom) $25 \%$ of genes can be found in the Supplementary File Section 4 for $15 \%$ (from Supplementary Figure 4-1 to Supplementary Figure 4-10 and from Supplementary Table 4-1 to Supplementary Table 4-4), and Section 5 for 25\% (from Supplementary Figure 5-1 to Supplementary Figure 5-10 and from Supplementary Table 5-1 to Supplementary Table 5-4).
In this study, considering the nonuniform of methylation levels along the gene region, we re-analyzed the relationship between the fluctuation of methylation levels and evolutionary conservation on 5 different DNA elements including exon, intron, 3'UTR, 5'UTR and upstream regions of the transcription start site (TSS1500). For each region, we found that the results still supported that the SM genes had higher evolutionary conservation than FM genes. The results for 5 different regions can be found in the Supplementary File Section 6 for exon (from Supplementary Figure 6-1 to Supplementary Figure 6-10 and from Supplementary Table 6-1 to Supplementary Table 6-4), Section 7 for intron (from Supplementary Figure 7-1 to Supplementary Figure 7-10 and from Supplementary Table 7-1 to Supplementary Table 7-4), Section 8 for 3'UTR (from Supplementary Figure 8-1 to Supplementary Figure 8-10 and from Supplementary Table 8-1 to Supplementary Table 8-4), Section 9 for 5'UTR (from Supplementary Figure 9-1 to Supplementary Figure 9-10 and from Supplementary Table 9-1 to Supplementary Table 9-4) and Section 10 for TSS1500 (from Supplementary Figure 10-1 to Supplementary Figure 10-10 and from Supplementary Table 10-1 to Supplementary Table 10-4). These results indicated that the nonuniform of methylation levels along the gene region had little effect on the analysis result.

As mentioned above, SM genes were defined as genes that have stable DNA methylation levels in all cell types of every tissue under normal or disease conditions. Therefore, all 8676 samples from the 108 series of GPL13534 were put together to form a large data set and used to compare the evolutionary conservation between SM and FM genes. To illustrate whether the disease condition could influence the results, we used a methylation dataset (GSE40699) which included 60 different normal cell lines from ENCODE Project and investigated the relationship between the stability of methylation status and evolutionary conservation. The results still supported the hypothesis that the SM genes had higher evolutionary conservation than FM genes (for details, see Supplementary File Section 11, from Supplementary Figure 11-1 to Supplementary Figure 11-10 and from Supplementary Table 11-1 to Supplementary Table 11-4). The consistency of the results suggested that the disease information did not affect the results. These tests indicated that our results are highly reliable.

By comparing of the evolutionary features between the SM genes and the FM genes, we got results and conclusions supporting that genes with stable DNA methylation levels show higher evolutionary conservation than genes with fluctuant DNA methylation levels. These results and conclusions may benefit for the following researches associated with the stability of methylation levels or evolutionary conservation. Based on these results, we could further explore the relationship between the stability 
of methylation levels and the stability of other omics such as genomics, transcriptome and proteomics. We also could try to investigate the relationship between the stability of methylation levels and complex diseases. Ultimately, we expect that our results will improve our understanding of the stabilizing mechanisms of biological systems.

\section{MATERIALS AND METHODS}

\section{DNA methylation data}

The DNA methylation data for human genes were obtained from the NCBI GEO database [24, 25]. We downloaded methylated and unmethylated signal data of all the 108 series from the GPL13534 platform (Illumina HumanMethylation450 BeadChip, including 485,577 probes). All the methylated and unmethylated signal values were normalized [26]. The total number of samples was 8,676 . For each sample, we used the -value to represent the methylation status at a probed location. The -value was defined as

, where is the methylated signal and is the unmethylated signal at a probed location and 100 is a constant for regularizing the beta value when both methylated and unmethylated probe intensities are low [27]. We then mapped the probes to genes based on the annotation information of the platform. The methylation level of a gene, was defined as the average -values of probes mapped to the gene. Considering the possible batch effect among different series, we performed adjustment for in different series using the Empirical Bayes (EB) batch correction method [28]. Ultimately, the DNA methylation data set included 21,231 genes and their DNA methylation levels in 8,676 samples.

\section{SM genes and FM genes}

For a gene, we use the variance of to measure the variations of DNA methylation levels. In this study, the variance of is also called the fluctuation coefficient, FC. The FC of a human gene is defined as:

where is the DNA methylation level of gene in the th sample, is the number of samples examined, and is the average DNA methylation level of gene across the samples. A higher value indicates larger variations in DNA methylation levels across samples, and a lower value indicates lower variations (or higher stability) in DNA methylation levels across samples. We then sorted the genes from low values at the top to highest values at the bottom. The top $20 \%$ of sorted genes are used as SM genes, and the bottom $20 \%$ of sorted genes are used as FM genes. Finally, we obtained 4,247 SM genes and 4,247 FM genes.

\section{Orthologous genes}

Orthologous genes of the human genes were downloaded from the Ensemble database (ftp://ftp.ensembl .org/pub/release-69/mysql/ensembl_mart_69) [29-31]. There were 21 species (Full names and abbreviations of the 21 species can be seen in Supplementary Table S5) which had non-null data. We extracted the one-to-one orthologous genes [32], and the dn (rate of non-synonymous substitutions) and ds (synonymous substitutions) values from the downloaded files of 21 species. For SM and FM genes, the percentage of orthologous genes for each species was calculated. For each pair of human-other species orthologous genes, we calculated the evolutionary rate $\mathrm{dn} / \mathrm{ds}$.

To calculate the sequence identity, we downloaded the protein sequences between pairwise human-other species orthologous genes from BioMart (http://www. ensembl.org/biomart/martview) [33, 34]. The BLASTP program and the BLOSUM62 matrix $[35,36]$ were used to align the orthologous sequences. The sequence identity was defined as the percent identity of the match.

\section{SNP data}

The SNP data were downloaded from the NCBI SNP database (http://www.ncbi.nlm.nih.gov/SNP/) [37]. The ID numbers (rs\#) and positions of the SNPs were extracted. We then downloaded the location information (start and end position) of SM and FM genes from NCBI (ftp://ftp .ncbi.nlm.nih.gov/genomes/MapView/), and mapped the SNPs to the SM and FM genes based on the chromosomal location. Finally, we calculated the SNP density (the number of SNPs divided by the length of the gene) for each SM or FM gene.

\section{The HapMap project data}

We used public genotype data of common SNPs from the HapMap project $[38,39]$. The raw data were downloaded from the NCBI HapMap website (ftp://ftp .ncbi.nih.gov/hapmap). 1,117 unrelated individuals from 11 global populations [40] were extracted from the raw data (Full names and abbreviations of the 11 HapMap populations can be seen in Supplementary Table S5). The SNPs included in this study passed the following quality control (QC) criteria: minor allele frequency (MAF) is greater than 0.01 , P-value of the HardyWeinberg equilibrium (HWE) test is greater than 0.001 , call frequency is greater than 0.75 , and the SNP must be detected in all 11 populations. We then mapped the SNPS to the SM and FM genes based on the location information, and calculated the pairwise linkage disequilibrium (LD) coefficient, $r^{2}$, for all the SNPs in an SM or FM gene 
region using the Haploview software [41]. The median pairwise $r^{2}$ in a gene region was calculated and compared between SM and FM genes.

\section{The 1000 genomes project data}

To verify our conclusions, we used another highquality data set, the 1000 genomes project [42], to compare the degree of LD between SM and FM genes. The raw genotype data were downloaded from the NCBI 1000 genomes website (http://ftp.ncbi.nlm.nih. gov/1000genomes/). 1,063 unrelated individuals from 14 global populations were extracted from the raw dataset (for details, see Supplementary Table S5). Only 13 populations were included in this study, because the IBS population only had 14 samples. The SNP genotype data of the 1000 genomes project were filtered based on the same QC criteria as the HapMap genotype data. Ultimately, the $\mathrm{r}^{2}$ for each of the SM and FM genes were calculated.

\section{Gene ontology (GO) annotation}

To better understand the biological reasons for the observed differences in evolutionary conservation, we compared the Gene Ontology (GO) of the SM and FM genes [43-45]. We used the DAVID software [46] to annotate the SM and FM genes. The biological process (BP) and molecular function (MF) were used to compare the functional differences between SM and SM genes. The cell component (CC) annotation results are also listed in Section 2 and 3 of the Supplementary File.

\section{Statistical analysis}

We used the Wilcoxon rank sum test to compare whether an evolutionary feature was significantly different between the SM and FM genes for each individual species or population. We used the Wilcoxon signed rank test [47] to test whether the median of an evolutionary feature was significantly different between the SM and FM genes across all species or populations. All data were processed using Perl scripts (http://www .activestate.com/activeperl). All statistical graphics and calculations were completed using R scripts (http:// cran.r-project.org). All the Perl scripts and R scripts can be found at the website: http://www.bioapp.org/research/ SMvsFM.

\section{ACKNOWLEDGMENTS}

This work was supported in part by grants from the National Natural Science Foundation of China (grant numbers 31200934, 61300116 and 81300945) and the Natural Science Foundation of Heilongjiang Province (grant numbers C201206 and QC2013C063).

\section{CONFLICTS OF INTEREST}

The authors declare that they have no conflict of interest.

\section{REFERENCES}

1. Michelotti GA, Brinkley DM, Morris DP, Smith MP, Louie RJ, Schwinn DA. Epigenetic regulation of human alpha1d-adrenergic receptor gene expression: a role for DNA methylation in Sp1-dependent regulation. FASEB J. 2007; 21:1979-1993.

2. Jones PA, Takai D. The role of DNA methylation in mammalian epigenetics. Science. 2001; 293:1068-1070.

3. Linn F, Heidmann I, Saedler H, Meyer P. Epigenetic changes in the expression of the maize A1 gene in Petunia hybrida: role of numbers of integrated gene copies and state of methylation. Mol Gen Genet. 1990; 222:329-336.

4. Shirodkar AV, St Bernard R, Gavryushova A, Kop A, Knight BJ, Yan MS, Man HS, Sud M, Hebbel RP, Oettgen P, Aird WC, Marsden PA. A mechanistic role for DNA methylation in endothelial cell (EC)-enriched gene expression: relationship with DNA replication timing. Blood. 2013; 121:3531-3540.

5. Li E, Bestor TH, Jaenisch R. Targeted mutation of the DNA methyltransferase gene results in embryonic lethality. Cell. 1992; 69:915-926.

6. Okano M, Bell DW, Haber DA, Li E. DNA methyltransferases Dnmt3a and Dnmt3b are essential for de novo methylation and mammalian development. Cell. 1999; 99:247-257.

7. Illingworth R, Kerr A, Desousa D, Jorgensen H, Ellis P, Stalker J, Jackson D, Clee C, Plumb R, Rogers J, Humphray S, Cox T, Langford C, Bird A. A novel CpG island set identifies tissue-specific methylation at developmental gene loci. PLoS Biol. 2008; 6:e22.

8. Heard E, Clerc P, Avner P. X-chromosome inactivation in mammals. Annu Rev Genet. 1997; 31:571-610.

9. Li E, Beard C, Jaenisch R. Role for DNA methylation in genomic imprinting. Nature. 1993; 366:362-365.

10. Reik W. Stability and flexibility of epigenetic gene regulation in mammalian development. Nature. 2007; 447:425-432.

11. Rakyan VK, Down TA, Thorne NP, Flicek P, Kulesha E, Graf S, Tomazou EM, Backdahl L, Johnson N, Herberth M, Howe KL, Jackson DK, Miretti MM, Fiegler H, Marioni JC, Birney E, et al. An integrated resource for genome-wide identification and analysis of human tissue-specific differentially methylated regions (tDMRs). Genome Res. 2008; 18:1518-1529.

12. Silva PN, Furuya TK, Braga IL, Rasmussen LT, Labio RW, Bertolucci PH, Chen ES, Turecki G, Mechawar N, Payao SL, Mill J, Smith MC. Analysis of HSPA8 and HSPA9 mRNA expression and promoter methylation 
in the brain and blood of Alzheimer's disease patients. J Alzheimers Dis. 2014; 38:165-170.

13. Silva PN, Furuya TK, Sampaio Braga I, Rasmussen LT, de Labio RW, Bertolucci PH, Chen ES, Turecki G, Mechawar N, Payao SL, Mill J, Smith MC. CNP and DPYSL2 mRNA expression and promoter methylation levels in brain of Alzheimer's disease patients. J Alzheimers Dis. 2013; 33:349-355.

14. Chen Y, Wu F, Liu M, Parvez F, Slavkovich V, Eunus M, Ahmed A, Argos M, Islam T, Rakibuz-Zaman M, Hasan R, Sarwar G, Levy D, Graziano J, Ahsan H. A prospective study of arsenic exposure, arsenic methylation capacity, and risk of cardiovascular disease in Bangladesh. Environ Health Perspect. 2013; 121:832-838.

15. Crescenti A, Sola R, Valls RM, Caimari A, Del Bas JM, Anguera A, Angles N, Arola L. Cocoa Consumption Alters the Global DNA Methylation of Peripheral Leukocytes in Humans with Cardiovascular Disease Risk Factors: A Randomized Controlled Trial. PLoS One. 2013; 8:e65744.

16. Zhang J, Xing B, Song J, Zhang F, Nie C, Jiao L, Liu L, Lv F, Wang S. Associated Analysis of DNA Methylation for Cancer Detection Using CCP-Based FRET Technique. Anal Chem. 2014; 86:346-350.

17. Xu X, Gammon MD, Hernandez-Vargas H, Herceg Z, Wetmur JG, Teitelbaum SL, Bradshaw PT, Neugut AI, Santella RM, Chen J. DNA methylation in peripheral blood measured by LUMA is associated with breast cancer in a population-based study. FASEB J. 2012; 26:2657-2666.

18. Marth GT, Korf I, Yandell MD, Yeh RT, Gu Z, Zakeri H, Stitziel NO, Hillier L, Kwok PY, Gish WR. A general approach to single-nucleotide polymorphism discovery. Nat Genet. 1999; 23:452-456.

19. Hinds DA, Stuve LL, Nilsen GB, Halperin E, Eskin E, Ballinger DG, Frazer KA, Cox DR. Whole-genome patterns of common DNA variation in three human populations. Science. 2005; 307:1072-1079.

20. DeBerardinis RJ, Thompson CB. Cellular metabolism and disease: what do metabolic outliers teach us? Cell. 2012; 148:1132-1144.

21. Metallo CM MG. Understanding metabolic regulation and its influence on cell physiology. Molecular cell. 2013; 49:388-398.

22. Cairns RA, Harris IS, Mak TW. Regulation of cancer cell metabolism. Nature Reviews Cancer. 2011; 11:85-95.

23. Saltiel AR, Kahn CR. Insulin signalling and the regulation of glucose and lipid metabolism. Nature. 2001; 414:799-806.

24. Edgar R, Barrett T. NCBI GEO standards and services for microarray data. Nat Biotechnol. 2006; 24:1471-1472.

25. Barrett T, Wilhite SE, Ledoux P, Evangelista C, Kim IF, Tomashevsky M, Marshall KA, Phillippy KH, Sherman PM, Holko M, Yefanov A, Lee H, Zhang N, Robertson CL, Serova N, Davis S, et al. NCBI, GEO archive for functional genomics data sets - update. Nucleic Acids Res. 2013; 41:D991-995.

26. Du P, Kibbe WA, Lin SM. lumi: a pipeline for processing Illumina microarray. Bioinformatics. 2008; 24:1547-1548.

27. Bibikova M, Lin Z, Zhou L, Chudin E, Garcia EW, Wu B, Doucet D, Thomas NJ, Wang Y, Vollmer E. Highthroughput DNA methylation profiling using universal bead arrays. Genome research. 2006; 16:383-393.

28. Leek JT, Johnson WE, Parker HS, Jaffe AE, Storey JD. The sva package for removing batch effects and other unwanted variation in high-throughput experiments. Bioinformatics. 2012; 28:882-883.

29. Hubbard T, Barker D, Birney E, Cameron G, Chen Y, Clark L, Cox T, Cuff J, Curwen V, Down T, Durbin R, Eyras E, Gilbert J, Hammond M, Huminiecki L, Kasprzyk A, et al. The Ensembl genome database project. Nucleic Acids Res. 2002; 30:38-41.

30. Flicek P, Amode MR, Barrell D, Beal K, Brent S, CarvalhoSilva D, Clapham P, Coates G, Fairley S, Fitzgerald S, Gil L, Gordon L, Hendrix M, Hourlier T, Johnson N, Kahari AK, et al. Ensembl. Nucleic Acids Res. 2012; 40:D84-90.

31. Flicek P, Amode MR, Barrell D, Beal K, Billis K, Brent S, Carvalho-Silva D, Clapham P, Coates G, Fitzgerald S, Gil L, Giron CG, Gordon L, Hourlier T, Hunt S, Johnson N, et al. Ensembl. Nucleic Acids Res. 2014; 42:D749-755.

32. Vilella AJ, Severin J, Ureta-Vidal A, Heng L, Durbin R, Birney E. EnsemblCompara GeneTrees: Complete, duplication-aware phylogenetic trees in vertebrates. Genome Res. 2009; 19:327-335.

33. Zhang J, Haider S, Baran J, Cros A, Guberman JM, Hsu J, Liang Y, Yao L, Kasprzyk A. BioMart: a data federation framework for large collaborative projects. Database (Oxford). 2011; 2011-bar038.

34. Kinsella RJ, Kahari A, Haider S, Zamora J, Proctor G, Spudich G, Almeida-King J, Staines D, Derwent P, Kerhornou A, Kersey P, Flicek P. Ensembl BioMarts: a hub for data retrieval across taxonomic space. Database (Oxford). 2011; 2011:bar030.

35. Altschul SF, Gish W, Miller W, Myers EW, Lipman DJ. Basic local alignment search tool. J Mol Biol. 1990; 215:403-410.

36. Altschul SF, Madden TL, Schaffer AA, Zhang J, Zhang Z, Miller W, Lipman DJ. Gapped BLAST and PSI-BLAST: a new generation of protein database search programs. Nucleic Acids Res. 1997; 25:3389-3402.

37. Sherry ST, Ward MH, Kholodov M, Baker J, Phan L, Smigielski EM, Sirotkin K. dbSNP: the NCBI database of genetic variation. Nucleic Acids Res. 2001; 29:308-311.

38. Frazer KA, Ballinger DG, Cox DR, Hinds DA, Stuve LL, Gibbs RA, Belmont JW, Boudreau A, Hardenbol P, Leal SM, Pasternak S, Wheeler DA, Willis TD, Yu F, Yang $\mathrm{H}$, Zeng $\mathrm{C}$, et al. A second generation human 
haplotype map of over 3.1 million SNPs. Nature. 2007; 449:851-861.

39. Altshuler DM, Gibbs RA, Peltonen L, Dermitzakis E, Schaffner SF, Yu F, Bonnen PE, de Bakker PI, Deloukas P, Gabriel SB, Gwilliam R, Hunt S, Inouye M, Jia X, Palotie A, Parkin M, et al. Integrating common and rare genetic variation in diverse human populations. Nature. 2010; 467:52-58.

40. Pemberton TJ, Wang C, Li JZ, Rosenberg NA. Inference of unexpected genetic relatedness among individuals in HapMap Phase, III. Am J Hum Genet. 2010; 87:457-464.

41. Barrett JC, Fry B, Maller J, Daly MJ. Haploview: analysis and visualization of LD and haplotype maps. Bioinformatics. 2005; 21:263-265.

42. Abecasis GR, Auton A, Brooks LD, DePristo MA, Durbin RM, Handsaker RE, Kang HM, Marth GT GA. An integrated map of genetic variation from 1,092 human genomes. Nature. 2012; 491:56-65.

43. Blake JA, Harris MA. The Gene Ontology (GO) project: structured vocabularies for molecular biology and their application to genome and expression analysis. Curr Protoc Bioinformatics. Chapter 7:Unit 7-22008.
44. Harris MA, Clark J, Ireland A, Lomax J, Ashburner M, Foulger R, Eilbeck K, Lewis S, Marshall B, Mungall C, Richter J, Rubin GM, Blake JA, Bult C, Dolan M, Drabkin $\mathrm{H}$, et al. The Gene Ontology (GO) database and informatics resource. Nucleic Acids Res. 2004; 32:D258-261.

45. Barrell D, Dimmer E, Huntley RP, Binns D, O'Donovan C, Apweiler R. The GOA database in - an integrated Gene Ontology Annotation resource. Nucleic Acids Res. 2009; 37:D396-403.

46. Huang da W Sherman, BT Tan, Q Kir, J Liu, D Bryant, D Guo, Y Stephens, R Baseler, MW Lane, HC and Lempicki. DAVID Bioinformatics Resources: expanded annotation database and novel algorithms to better extract biology from large gene lists. Nucleic Acids Res. 2007; 35:W169-175.

47. Wilcoxin F. Individual comparisons by ranking methods. Biometrics. 1945; 1:80-83. 